\title{
LAS BIBLIOTECAS UNIVERSITARIAS ARGENTINAS Y LA EDUCACIÓN EN LÍNEA ANTE LA PANDEMIA POR COVID19
}

\author{
Anabel Gaitán* \\ Facultad de Humanidades. Universidad Católica de Santa Fe. \\ María Inés Coraglia** \\ Universidad Católica de Santa Fe
}

\begin{abstract}
Resumen: Nos propusimos evaluar los procesos de innovación y cambio ocurridos en las bibliotecas universitarias de la Argentina en ocasión de la pandemia, identificando para ello el porfolio de servicios que mantuvieron las mismas, sus modificaciones y reconociendo además las diferentes metodologías de comunicación y gestión internas y con la comunidad de usuarios en contextos de aislamiento.

Realizamos un relevamiento de información documental a través de los sitios web de las bibliotecas y las organizaciones que agrupan el colectivo bibliotecario, con el fin de evaluar y comparar las respuestas de estas ante la crisis a nivel nacional (Argentina), y de pares en Iberoamérica. Luego se aplicó un instrumento de recolección de información directa al conjunto de 326 bibliotecas pertenecientes a las 131 universidades argentinas. Finalmente arribamos a conclusiones que nos permiten ponderar la identidad de las bibliotecas universitarias en la educación superior post COVID19.
\end{abstract}

Palabras clave: Biblioteca universitaria; educación superior; bibliotecología; educación online; aprendizaje virtual.

Title: ARGENTINE UNIVERSITY LIBRARIES AND ONLINE EDUCATION IN THE FACE OF THE COVID19 PANDEMIC.

Abstract: We set out to evaluate the processes of innovation and change that occurred in the University Libraries of Argentina during the pandemic, identifying the portfolio of services that they maintained, their modifications and also recognizing the different internal communication and management methodologies and with the user community in isolation contexts.

We carry out a survey of information, documentary through the websites of the Libraries and the organizations that make up the Librarian collective, in order to evaluate and compare their responses to the crisis at the national level (Argentina), and peers in Iberoamerica. Then a direct information collection instrument was applied to the set of 326 Libraries belonging to the 131 Argentine Universities. Finally, we arrive at conclusions that allow us to ponder the identity of University Libraries in Post-COVID19 Higher Education.

Keywords: Academic library; higher education; librarianship; online education; virtual learning.

Copyright: (C) 2021 Servicio de Publicaciones de la Universidad de Murcia (Spain). Este es un artículo de acceso abierto distribuido bajo los términos de la licencia Creative Commons Reconocimiento 4.0 Internacional (CC BY 4.0).

\section{INTRODUCCIÓN}

Resulta necesario partir de considerar a las bibliotecas universitarias y su evolución en función de las crecientes necesidades de la comunidad universitaria antes de posicionarnos en el análisis del ahora. En este sentido, entre las diferentes conceptualizaciones que al respecto de este tema se van desarrollando, acordamos con la idea vertida hace ya dos décadas que entiende que "la biblioteca universitaria es un servicio de vital importancia para las universidades, puesto que es una combinación orgánica de personas, recursos, colecciones locales y virtuales, infraestructura, cuyo propósito es apoyar a los usuarios, en el proceso de transformar la información en conocimiento". (Comisión Asesora de Bibliotecas y Documentación - COBID, 2001)

La importancia del concepto seleccionado, más allá del tiempo transcurrido desde su definición, está dada por la utilización del término combinación (definida como mezcla o unión de personas o cosas distintas que componen un todo o la forma de estar combinadas o coordinadas cosas, personas o acciones de forma que se favorece el funcionamiento o desarrollo de algo) reforzada por la característica de orgánica (en su acepción adjetiva cuando considera a un ente constituido por unidades que forman un conjunto organizado u ordenado). Además, porque también se hace referencia al acceso presencial y remoto de la información (locales y virtuales) y a su propósito de apoyar a los usuarios a transformar la información en conocimiento, es decir, todas las actividades que suponen no

\footnotetext{
*agaitan@ucsf.edu.ar

**micoraglia@ucsf.edu.ar
} 
solo acceder a los hechos, sino transformarlos en comprensión sobre la naturaleza de los mismos, sus relaciones y cualidades; siendo esto último una facultad eminentemente humana, y adelantándose a acciones que hoy mismo le son requeridas a las bibliotecas universitarias.

Así, las bibliotecas universitarias como parte significativa del sistema de educación superior evolucionan adaptándose a las nuevas necesidades de sus comunidades de usuarios, incluso llegando a atender las nuevas maneras de enseñar y aprender. Así, Brown las llama espacios de aprendizaje de próxima generación (Brown, Bennett, Henson y Valk, 2014) y Gallo León (2015) postula que las bibliotecas han progresado de ser "sitios para consumir cosas a sitios donde hacer cosas" (p. 89), destacando de esta forma su nuevo papel en el fomento del aprendizaje autónomo, y en la misma línea, Watson (2017) analiza los cambios que se producen actualmente en la sociedad, el aprendizaje y la tecnología; y que se pueden tener en cuenta a la hora de diseñar las bibliotecas universitarias y prepararlas para el siglo XXI, fomentando y apoyando el aprendizaje fuera del aula.

UNESCO (2020) da cuenta de que el 60.5\% de los alumnos matriculados en el mundo (en todos los niveles) se han visto afectados por alteraciones en sus rutinas educativas formales por la crisis COVID19. Focalizando la mirada en estudios terciarios (universitarios), en España por ejemplo más de 300.000 estudiantes se vieron afectados. En Perú la cifra asciende a 1,8 millones, en Colombia a 2,4 millones, en Argentina a 3,1 millones y en México supera los 4 millones.

La Coalición Internacional de Consorcios de Bibliotecas conformada por 200 miembros de los cinco continentes pertenecientes a todo tipo y tamaño de bibliotecas, emite una declaración en marzo de 2020 a través de la que ponen de manifiesto la preocupación ante el colectivo de editoriales de textos académicos y científicos para que el acceso a la información sea posible en modalidad online remota en forma total.

Por su parte, la Red de Bibliotecas de las Universidades Españolas (2020) a golpe de vista en su sitio web nos pone de lleno en el tema de la crisis por pandemia, y a pocos días de declarada la misma en el mes de marzo de 2020, emite una declaración a favor del conocimiento abierto y sostenible, reconociendo la importancia de compartir el conocimiento científico de forma inmediata y abierta a la comunidad que investiga; facilitando el acceso y la circulación del mismo en beneficio de otros a nivel mundial, y aunque destacan los avances del movimiento ciencia abierta, reconocen que aún hay "muros económicos" vigentes; y un par de meses después publican junto a otras tres organizaciones vinculadas a la edición y comercialización de información científica, un comunicado que expresa el compromiso de todos para el mantenimiento de las colecciones de información científica en lo inmediato y frente a la crisis económica que se avecina en la pospandemia.

Por su parte, la Asociación Colombiana de Universidades (2020) publica un documento en el que a partir de ejemplos de lo que 35 universidades colombianas han venido realizando en este corto período de crisis, buscan generar información de valor coyuntural para todas las instituciones de educación superior del país.

La RedIAB, Red Interuniversitaria Argentina de Bibliotecas (2020) y la red Amicus, Red de Bibliotecas de Universidades Privadas (2020), no ofrecen información de acceso público a través de sus sitios web sobre acciones a seguir al respecto de cómo actuar en el contexto de la pandemia por COVID19, sin embargo, en las webs de las universidades que son miembro de estas redes si es posible visualizar diferentes informaciones sobre este tema.

En los Estados Unidos, la Comisión Directiva de la Asociación de Bibliotecas Universitarias y de Investigación ACRL oficialmente instó formalmente a todos sus miembros a cerrar completamente los servicios presenciales y reemplazar los accesos restringidos online por accesos libres para la continuidad del trabajo.

\section{OBJETIVOS}

Nos proponemos evaluar los procesos de innovación y cambio en ocasión de la pandemia. Consecuentemente, planteamos identificar el porfolio de servicios de las bibliotecas universitarias con especial foco en Argentina, sus modificaciones frente a la complejidad causada por la pandemia COVID19, reconociendo las diferentes metodologías de comunicación y gestión (internas y con su comunidad de usuarios) en contextos de aislamiento, así como la modificación de la propia identidad de las bibliotecas universitarias a futuro. 


\section{METODOLOGÍA}

La investigación es de índole cuanti-cualitativa y pretende la descripción de la situación actual de la población estudiada.

Realizamos un relevamiento de información documental a través de sitios web de bibliotecas académicas y otras organizaciones que agrupan el colectivo bibliotecario vinculado al ámbito universitario, tanto a nivel nacional (Argentina), como de pares en Iberoamérica y a través de otros actores internacionales de reconocido prestigio. Este proceso tuvo como fin evaluar y comparar las respuestas de estas ante la crisis sanitaria por COVID19, a fin de construir un marco teórico de referencia en la cuestión.

En segundo lugar, nos abocamos a la construcción de un instrumento de recogida de información que aplicamos en forma directa al conjunto de 326 bibliotecas pertenecientes a las 131 universidades argentinas, para conocer de primera mano la situación de las mismas en estos cuatro meses de pandemia.

Se obtuvieron 74 respuestas provenientes de 51 universidades (39\% del total de instituciones); las que fueron respondidas en su mayoría por directores de la biblioteca (57\%) o personal con formación en bibliotecología (39\%).

\section{RESULTADOS}

Ante la consulta sobre la modalidad de trabajo adoptada, resulta muy importante destacar que el $100 \%$ manifiesta haber continuado trabajando de uno u otro modo. Lidera ampliamente la adopción del home office, es decir el desarrollo equivalente de las tareas y actividades habituales, pero sin presencia física en las bibliotecas. Solo el 13\% refiere continuar realizando algún tipo de actividades en las mismas.

\begin{tabular}{|l|c|c|}
\hline \multicolumn{2}{|c|}{ ¿Qué modalidad de trabajo han adoptado? } & $80 \%$ \\
\hline Home office (sin presencia física alguna en la biblioteca). & 59 & $5 \%$ \\
\hline Con personal presente en el lugar, pero sin atención al público. & 4 & $8 \%$ \\
\hline Con personal presente en el lugar y atención al público en forma restringida. & 6 & $0 \%$ \\
\hline Habitual, sin restricciones. & 0 & $7 \%$ \\
\hline Otras & 5 & $100 \%$ \\
\hline Total & 74 & $00 \%$ \\
\hline
\end{tabular}

Tabla I. Modalidad de trabajo adoptada por las bibliotecas universitarias. Fuente: elaboración propia.

En relación con las estrategias y modalidades de comunicación entre los miembros del equipo de trabajo, se destaca la utilización de Whatsapp ( $92 \%$ de las bibliotecas), seguido por el correo electrónico ( $85 \%$ de las mismas). Resulta además relevante rescatar la adopción de Zoom y Meet como herramientas de videoconferencia (en ambos casos referidas por más del 30\% de los encuestados), y otras acciones menos nombradas como el uso de Jitsi, Google Calendar y Telegram.

\begin{tabular}{|l|c|c|}
\hline \multicolumn{3}{|c|}{ ¿Cómo se realiza la comunicación? } \\
\hline Whatsapp & 68 & $92 \%$ \\
\hline Email & 63 & $85 \%$ \\
\hline Zoom & 26 & $35 \%$ \\
\hline Meet & 24 & $32 \%$ \\
\hline Otras & 14 & $19 \%$ \\
\hline Presencial & 6 & $8 \%$ \\
\hline Telegram & 1 & $1 \%$ \\
\hline
\end{tabular}

Tabla II. Metodología de comunicación de los equipos de trabajo. Fuente: elaboración propia. 
En relación con los medios de comunicación y acceso de los estudiantes a la biblioteca, se mantiene la tendencia de la alta adopción de Whatsapp, correo electrónico e Instagram (86\% de las bibliotecas), seguido por la utilización de formularios online (en el $47 \%$ de los casos) y el campus virtual (en el $41 \%$ de los casos).

\begin{tabular}{|l|c|c|}
\hline \multicolumn{3}{|c|}{ ¿Cómo acceden durante esta etapa los estudiantes a la biblioteca? } \\
\hline Whatsapp /email/ Instagram & 64 & $86 \%$ \\
\hline Formulario online & 35 & $47 \%$ \\
\hline Campus virtual & 30 & $41 \%$ \\
\hline Otras & 25 & $34 \%$ \\
\hline Meet & 7 & $9 \%$ \\
\hline Presencial & 7 & $9 \%$ \\
\hline Zoom & 5 & $7 \%$ \\
\hline Telegram & 0 & $0 \%$ \\
\hline
\end{tabular}

Tabla III. Metodología de comunicación con los usuarios. Fuente: elaboración propia.

Una de las cuestiones sobresalientes de la consulta, fue la vinculada al tipo de servicios para alumnos que cada biblioteca pudo sostener o incorporar durante este período tan especial y así, más del 90\% de los consultados destacó el acceso a base de datos; el $64 \%$ hizo lo propio con el servicio de asistencia técnica remota y el $49 \%$ mencionó la posibilidad de ofrecer capacitación. Por otra parte, la capacitación en Alfabetización Informacional fue mencionado por el 38\% y el 34\% nombró otras actividades, entre las que se destacan el servicio de referencia y búsqueda de bibliografía (15\%) y la adquisición y préstamo de ebooks $(10 \%)$. Con menor incidencia fueron nombrados el acceso a recursos y objetos de aprendizaje (fichas de cátedra, presentaciones en diapositivas, guías de lecturas realizadas por profesores entre otros) y el asesoramiento para la escritura de tesis. Se nombraron además de la realización de "maratón de lectura"; la aplicación de estrategias que "se promueven y acompañan las actividades de capacitación organizadas desde la dirección general del sistema"; el "apoyo a las actividades de enseñanza a distancia a través de consultas por mail" y "préstamo de libros a domicilio, envíos de jurisprudencia y doctrina vía correo electrónico y la liberación de los accesos a las bases de datos jurídicas".

\begin{tabular}{|l|c|c|}
\hline \multicolumn{3}{|c|}{ ¿Qué servicios se mantienen accesibles a los estudiantes? } \\
\hline Acceso a bases de datos & 69 & $93 \%$ \\
\hline Asistencia técnica & 47 & $64 \%$ \\
\hline Capacitación & 36 & $49 \%$ \\
\hline Alfin & 28 & $38 \%$ \\
\hline Otras & 25 & $34 \%$ \\
\hline Préstamos físicos & 11 & $15 \%$ \\
\hline
\end{tabular}

Tabla IV. Servicios mantenidos. Fuente: elaboración propia.

En comparación, la situación del colectivo de bibliotecas españolas, de acuerdo con los datos recopilados por la Rebiun y citados por Arroyo-Vázquez y Gómez-Hernández (2020) permiten afirmar que en un primer momento se han mantenido los servicios no presenciales y la atención al usuario por teléfono, correo electrónico y mensajería. Los períodos de préstamo se han ampliado automáticamente y se han incrementado los esfuerzos en la difusión de las colecciones electrónicas.

Con respecto a la pregunta abierta referida a la incorporación de nuevas tareas, actividades o servicios para atender las necesidades de profesores, investigadores y/o estudiantes durante la situación de aislamiento, se realizó una categorización respecto a las respuestas particulares de cada biblioteca.

Al respecto, de las 74 respuestas obtenidas, el 12\% manifiestan nulas o intrascendentes modificaciones en sus tareas, actividades o servicios, es decir que podemos afirmar el $88 \%$ de las bibliotecas adaptó su accionar a los desafíos planteados por el COVID19.

Del mismo modo, continuando la comparación con España, la Rebiun recomienda incentivar los servicios virtuales, dando prioridad a la adquisición de libros y revistas en formato digital, favoreciendo el préstamo electrónico, 
potenciando consultas y trámites online, consolidando la formación virtual, promoviendo la creación de materiales formativos y facilitando la digitalización de contenidos impresos (Arroyo-Vázquez y Gómez-Hernández, 2020). Coincidentemente con la información provista por Alonso Arévalo (2020) que plantea que los préstamos de libros electrónicos en bibliotecas vieron un incremento inédito, ya que a modo de ejemplo se expone la situación de las bibliotecas del Reino Unido, quienes manifestaron un crecimiento de un 358\% durante la cuarentena.

En este sentido, para organizar la información recabada respecto de las tareas, actividades o servicios incorporados durante el período actual, se segmentó el análisis respecto a los destinatarios (alumnos, docentes e investigadores) y a las acciones propiamente dichas. En función de las tareas, actividades y servicios se obtuvieron los siguientes resultados:

\begin{tabular}{|l|c|c|}
\hline \multicolumn{3}{|c|}{ Tareas, actividades y servicios } \\
\hline Virtualización de servicios & 11 & $15 \%$ \\
\hline Búsqueda de información & 13 & $18 \%$ \\
\hline Envío de información & 14 & $19 \%$ \\
\hline Integración con aula virtual & 4 & $6 \%$ \\
\hline Digitalización & 7 & $10 \%$ \\
\hline Asesoramiento & 12 & $17 \%$ \\
\hline Capacitación & 16 & $22 \%$ \\
\hline Recursos digitales & 25 & $35 \%$ \\
\hline
\end{tabular}

Tabla V. Tareas, actividades y servicios incorporados. Fuente: elaboración propia.

Con respecto a las tareas y servicios, existe una clara concentración en el fomento de repositorios y recursos digitales variados (entre los nombrados recursos bibliográficos de texto completo y recursos didácticos como atlas de anatomía en 3D de acceso remoto, editoriales y bibliotecas que han liberado su contenido: eLibro, Leer en casa, UNAM, Springer y editoriales propias de las universidades). También se denota que las bibliotecas han aprovechado la situación actual para generar propuestas de capacitación sincrónicas o asincrónicas ("Videos tutoriales de clases de capacitación sobre diversidad de temas que antes de la pandemia eran presenciales"; "también comenzamos a realizar capacitaciones online y webinars"; "capacitación en cómo desarrollar búsquedas en buscadores, metabuscadores, bases de datos, repositorios, etc."; "realizamos capacitaciones en línea"; "creamos un curso en línea sobre gestores bibliográficos entre otros"; "se incorporaron videoconferencias donde se capacitó sobre el acceso y búsquedas en el OPAC, uso de bases de datos").

Otros de los tópicos que fueron indagados se enfoca en la relación de las bibliotecas con docentes e investigadores y analizando los datos obtenidos, resulta destacable que en el $81 \%$ de los casos, la vinculación se mantuvo o se incrementó (47\% y 34\% respectivamente), mientras que el 7\% manifestó que disminuyó el intercambio y el 12\% expresó que la biblioteca no cuenta con los recursos para responder a las necesidades y requerimiento de docentes e investigadores.

\begin{tabular}{|c|c|c|}
\hline \multicolumn{3}{|c|}{ La relación entre docentes e investigadores y los bibliotecarios, a consecuencia de la pandemia } \\
\hline Se volvió más estrecha, nos apoyamos mutuamente. & 25 & $34 \%$ \\
\hline Se mantuvo, solo cambió el medio de comunicación, aunque es esporádica. & 35 & $47 \%$ \\
\hline $\begin{array}{l}\text { Disminuyó, y es fundamentalmente unidireccional: desde la biblioteca generamos } \\
\text { espacios y actividades para docentes que no siempre son aprovechados. }\end{array}$ & 5 & $7 \%$ \\
\hline $\begin{array}{l}\text { Los docentes solicitan apoyo a la biblioteca, que no siempre podemos brindarles (por } \\
\text { falta de recursos, tecnología y otras circunstancias). }\end{array}$ & 9 & $12 \%$ \\
\hline
\end{tabular}

Tabla VI. Caracterización de la relación entre docentes e investigadores y bibliotecarios. Fuente: elaboración propia.

Al momento de ampliar o fundamentar su respuesta anterior, las bibliotecas que manifiestan que la comunicación disminuyó sostienen que los servicios generados son aprovechados de manera muy desigual por los usuarios, otros de los inconvenientes nombrados tienen que ver con que los canales de distribución seleccionados no cumplen con las expectativas o los usuarios no cuentan con la capacitación para aprovecharlos. Otras de las cuestiones adicionales aducidas son las condiciones institucionales que exceden el ámbito de la biblioteca o la escasez de material digital, lo que dificulta poder satisfacer las nuevas necesidades de los usuarios. 
Con respecto a las bibliotecas que afirman que los docentes solicitan apoyo, y que esta situación que no siempre puede ser resuelta desde la misma biblioteca, las causas alegadas tienen que ver con el acceso a bases de datos restringido solo desde el equipamiento informático de la biblioteca; ausencia de presupuesto para acceder a bibliografía que no posee acceso libre (revistas de pago); carencias en el acceso al material de cátedra en formato digital; no contar con una base de ebooks; así como las limitantes del servicio de internet particular del personal de la biblioteca que dificulta su actividad. Otras de las causas nombradas tienen que ver con la amplitud de requerimientos y la imposibilidad de cubrirlos todos, por lo que una estrategia con la que se intenta suplir es a través de la cooperación interbibliotecaria.

Analizando las respuestas de aquellas bibliotecas que afirmaron que la comunicación se mantuvo, aunque esporádica, se destaca la utilización del email como medio de vinculación, o las estrategias de capacitación a través de la implementación de programas de Alfabetización Informacional (ALFIN) online. Además, resulta relevante reproducir a continuación algunas de las expresiones recogidas, que tiene que ver fundamentalmente con justificar el carácter esporádico de la relación, en función a la falta de interés o conocimiento de docentes e investigadores o a la carencia de recursos de la biblioteca:

\section{Falta de interés o conocimiento de los docentes/investigadores}

"Desde la biblioteca se generan contenidos y se ofrecen recursos que la mayoría de los docentes y alumnos no consulta. En este sentido, la presencialidad aún sigue marcando el ritmo de la biblioteca. Considero que se debe al desconocimiento de la gran cantidad de material digital disponible".

"Los docentes e investigadores no se acercan a la biblioteca, o por lo menos como lo esperamos y necesitamos los bibliotecarios. Muchas veces son los alumnos quienes nos informan sobre el material bibliográfico que les piden para avanzar en sus estudios".

"Docentes e investigadores desconocen el material ya sea papel o digital con que cuenta la biblioteca. Sí, hay excepciones, que colaboran, solicitan material, están informados sobre el material existente, pero son muy pocas".

"La comunicación entre los docentes e investigadores, en relación con los bibliotecarios es esporádica. Solamente se comunican vía e-mail, al servicio de referencia u otro sector cuando precisan solicitar información, cuando inician sus investigaciones".

"Recibimos pocas consultas de profesores y ninguna de investigadores, a pesar de que realizamos búsqueda de bibliografía digital para las materias con las que están trabajando no recibimos respuestas ni sugerencias al respecto".

"La relación con docentes e investigadores se da con pocas y particulares personas y debido a la falta de vínculo personal se vio especialmente afectada”.

"Es esporádica por la tarea que tienen los docentes para dar clases virtuales".

“Son pocos los docentes que utilizan servicios habituales, esto no se modificó durante la pandemia”.

\section{Carencia de recursos de las bibliotecas}

"La relación se mantuvo, aunque en ocasiones nos solicitan material al cual no tenemos acceso".

"El impedimento de la ley de derechos de autor hace que no podamos ofrecer el material impreso en formato digital y esto lleva a que el docente o el alumno lo consiga por vías no legales. En estos casos la biblioteca queda relegada".

“Algunas veces se solicita material digitalizado que no poseemos, el que quizás se encuentra en la plataforma bimodal a la que no tenemos acceso".

"Con algunos docentes se acrecentó con otros disminuyó. La consulta en sala que era muy frecuente por parte de tesistas e investigadores se ha visto totalmente resentida. El edificio está cerrado".

Tabla VII. Expresiones vinculadas a la esporádica o insuficiente comunicación entre docentes/investigadores y bibliotecarios. Fuente: elaboración propia. 
Finalmente, el 34\% de las bibliotecas afirmaron que la relación se volvió más estrecha, y estas sostienen la importancia del trabajo en conjunto de la comunidad académica para resolver los retos de la pandemia "La universidad tuvo que modificar su forma de evaluar y la biblioteca ayudó mucho con contenidos para usar en los exámenes y trabajos prácticos finales: balances, fallos, legislación, papers, casos de estudio, simuladores, etc.”. Por otro lado, se destaca la importancia de los canales seleccionados que facilitan el acceso de la comunidad académica a los servicios y recursos de las bibliotecas "La biblioteca propició un mayor acercamiento a los docentes", "la biblioteca ha llegado a más docentes, que a los que llegaba en forma presencial"; "el entorno virtual ha hecho que más docentes se contacten con nosotros. Aunque hay que seguir insistiendo un poco más".

También se hace manifiesta una revalorización del espacio, manifestando que las bibliotecas se encontraban preparadas para la virtualidad ("la biblioteca se mostró preparada para la educación en la virtualidad"; "se pudo visibilizar esto y poner en valor nuestro conocimiento relacionado a ambientes híbridos o virtuales") y destacándose como un espacio privilegiado de apoyo a la virtualidad "la biblioteca virtual es un aliado importante del campus virtual y van sumándose docentes a su uso" y de capacitación "al ofrecerles cursos y capacitación, la comunicación se volvió más fluida, y sobre todo valoran más la actividad de la biblioteca".

Respecto al rol de la biblioteca y su personal, en el proceso de enseñanza aprendizaje, puede visualizarse una dispersión de respuestas, ya que las opciones antagónicas poseen casi idénticos porcentajes de respuesta: la ausencia de integración (31\%) y la estrecha colaboración (32\%) y entre estas un tercer grupo que suma el $27 \%$ marcando una posición intermedia ("el equipo de biblioteca ofrece apoyo a la docencia e investigación. Igualmente, se percibe falta de integración"; "la integración se está dando muy lentamente"; "estamos trabajando en la integración de recursos en el campus virtual con miras al inicio del próximo cuatrimestre"), o con algún grado de integración parcial ("si bien mantenemos el repositorio de apuntes, no se percibe una integración activa respecto del campus virtual" o "con algunas carreras sí").

\begin{tabular}{|l|c|c|}
\hline \multicolumn{2}{|c|}{ ¿Considera que la biblioteca se encuentra integrada con las propuestas virtuales de enseñanza? } \\
\hline No, no se percibe integración. & 23 & $31 \%$ \\
\hline Existe integración de recursos. & 15 & $20 \%$ \\
\hline \begin{tabular}{l|c|} 
Existe integración de personal. \\
Los bibliotecarios colaboramos en el desarrollo de material didáctico, formación en \\
competencias digitales, asesoramiento, etc.
\end{tabular} & 5 & $3 \%$ \\
\hline Otras & 7 & $9 \%$ \\
\hline
\end{tabular}

Tabla VIII. Integración de la biblioteca con las propuestas de enseñanza virtuales. Fuente: elaboración propia.

Finalmente, se invitó a los consultados a expresar sus ideas abiertamente y a continuación incluimos un extracto de las recibidas, entre las que podemos destacar los conceptos de: crisis, oportunidad, accesibilidad, resistencia al cambio, adaptación, integración y acceso abierto. 
Siempre de las crisis sale algo positivo y esta pandemia hizo que se vean las falencias y que nos avoquemos a salvarlas.

Me parece excelente el momento de realizar la encuesta, ya que creo que la necesidad ha puesto en valor nuestros servicios.

Los bibliotecarios aún sin recursos, tenemos la gran posibilidad de re-inventarnos y mantenernos cerca de nuestra comunidad, aún en situaciones extremas e inesperadas como lo es esta pandemia.

Es necesaria la modificación de la Ley 11.723 de nuestro país, para poder avanzar con la accesibilidad al conocimiento en formatos digitales.

Me parece importante destacar que las restricciones sanitarias actuales nos obligaron a todos a utilizar recursos que ya teníamos pero que por inercia no queríamos utilizar. Esto se capitalizará en un futuro pues ya no habrá tanta resistencia al cambio dado que se tuvieron que realizar forzosamente.

La pandemia modificó la manera de consumir la información, estamos en procesos de cambios, y de adaptación al contexto. La biblioteca universitaria debe atender las nuevas necesidades de sus usuarios, percibir lo que sucede en este período y acompañar con innovación la disrupción.

Aprovechamos este tiempo para realizar trabajo interno (actualización del sistema de gestión, revisión de registros, puesta en funcionamiento de repositorio) lo que conlleva mucho tiempo. Si no estuviéramos en cuarentena no lo podríamos llevar adelante.

Necesidad que las bibliotecas puedan tener acceso a las distintas bases abiertas sobre todo las que pertenecen al Estado. Lo positivo son los cursos virtuales abiertos que ofrecen las bibliotecas organizadas. Especificas del área. Sugerencia desde las carreras que haya un plan de formación común para apoyar y direccionar el trabajo de las bibliotecas en tiempo de pandemia.

La pandemia es una oportunidad de percibir la necesidad de potenciar las colecciones virtuales y las interacciones medidas por tecnologías. El aprendizaje remoto es una realidad que llegó para quedarse y la biblioteca tiene que adaptar su paradigma a esta modalidad.

Tabla IX. Libre expresión. Fuente: elaboración propia.

\section{DISCUSIÓN}

El estudio precedente nos permitió sistematizar información para analizar las metodologías y estrategias de comunicación interna y externas de las bibliotecas universitarias, así como el sostenimiento de sus servicios y su relación con la comunidad de usuarios. A su vez analizamos los procesos de innovación que las mismas fueron generando a partir de enfrentarse a las situaciones que se les iban presentando. La conjunción de las estrategias de comunicación empleadas, los servicios sostenidos y los procesos de innovación generados nos permitirán concluir en las posibilidades de construcción de una identidad pospandemia.

\subsection{Metodologías de comunicación y gestión}

La metodología adoptada en el contexto de aislamiento se corresponde con el trabajo en la modalidad home office, es decir sin presencia física en la biblioteca, utilizando como medios de comunicación el Whatsapp, correo electrónico y las plataformas de videoconferencias. En pocas instituciones se consideró que la comunicación fue insuficiente o débilmente organizada.

Consecuentemente se consideraron los medios para el contacto con los usuarios (docentes, estudiantes e investigadores), destacándose las redes sociales (Whatsapp, Instagram), los correos electrónicos, los formularios online y el campus virtual.

La incorporación o refuerzo de estos medios de comunicación mediados por tecnología permitió que se mantengan o incorporen servicios y actividades que serán analizados a continuación, pero se puede concluir que la vinculación remota entre los miembros del equipo de trabajo de la biblioteca universitaria y/o con los usuarios de la misma, no solo no planteó mayores inconvenientes, sino que se sostuvo con actividad prácticamente en el total de las universidades.

\section{2 Porfolio de servicios}

Con respecto a los servicios mantenidos durante el periodo de aislamiento, se destacó el acceso a bases de datos (con un significativo 93\%), la asistencia técnica (64\% de las bibliotecas) y la posibilidad de capacitación (en la mitad de las instituciones); todas en modalidad remota. De lo anterior se observa un reforzamiento del rol de la biblioteca dentro de la universidad como proveedora de información, aunque la fuerte capacitación en alfabetización informacional comienza a mostrar su rol como un espacio de desarrollo para las competencias digitales docentes. 
Asimismo, con respecto a la incorporación de nuevas tareas o actividades, alrededor del 90\% de las bibliotecas manifiestan haber actuado en consecuencia dando cuenta de los cambios realizados.

Hay cuatro servicios que aparecen con mayor frecuencia. En primer orden la incorporación de todos los recursos científicos digitales de acceso abierto posibles; luego el concepto de curación de documentos, refiriendo a acciones tales como: ayuda en la búsqueda de información digital; selección de materiales digitales por especialidad; y, análisis de validez y legalidad de materiales bibliográficos online. En tercer lugar, la incorporación de la atención online por video llamadas y chat. Y finalmente en cuarto lugar el desarrollo de video tutoriales y webinars para asistir en la capacitación de coyuntura.

En referencia concreta a los servicios adicionados, se puede afirmar entonces, una fuerte concentración en el rol de proveedor de información (búsqueda, envío, digitalización, recursos digitales) mientras que solo el 6\% plantea una integración con los espacios virtuales de enseñanza y el $22 \%$ hace lo propio con las actividades relacionadas a la capacitación por lo que sigue siendo baja.

De esta forma, puede concluirse que la actual situación generó una reacción positiva en las bibliotecas universitarias, que fundamentalmente generó una traducción al medio digital/virtual del rol tradicional de las mismas.

\section{3 Procesos de innovación}

En líneas generales, cercano a la mitad de los encuestados sostiene que la relación entre docentes e investigadores y bibliotecarios durante este período de aislamiento y distanciamiento por pandemia se mantuvo igual, esporádica, reconociendo que solo cambió el medio de comunicación hacia un contacto virtual. Con respecto a las razones de esta situación, se destaca la utilización del email como medio de vinculación, la posibilidad de implementación de estrategias de ALFIN online y otras manifestaron que ya se encontraban trabajando en forma remota con docentes e investigadores. Resulta relevante destacar que algunas de las expresiones recogidas, tienen que ver fundamentalmente con justificar el carácter esporádico de la relación, en función a la falta de interés o conocimiento de docentes e investigadores o a la carencia de recursos de la biblioteca.

A esta percepción se suma más de un 30\% que reconoce un estrechamiento en la relación, a partir del mutuo apoyo frente a la situación inesperada y adversa. En general, advierten un reconocimiento a la tarea de las bibliotecas y una puesta en valor de los servicios que estas ofrecen a la comunidad educativa. Por otra parte, se destaca la importancia del trabajo en conjunto de la comunidad académica para resolver los retos de la pandemia, la importancia de los canales seleccionados que facilitan el acceso de la comunidad académica a los servicios y recursos de las bibliotecas, lo cual también se hace manifiesto en una revalorización del espacio, manifestando que las bibliotecas se encontraban preparadas para la virtualidad y destacándose como una plataforma privilegiada de apoyo a la virtualidad y de capacitación.

Un 12\% reconoce que la relación podría mejorar en la medida que las bibliotecas pudieran dar respuesta a ciertos pedidos que realizan los docentes y que por diferentes razones no es posible atender y describen a la razón del problema en cuestiones institucionales. Un pequeño porcentaje menor al $10 \%$ expresa que desde la biblioteca se generan espacios y actividades para docentes que no siempre son aprovechados.

Por otra parte, respecto al rol de la biblioteca y su personal en el proceso de enseñanza aprendizaje, se consultó respecto al grado de integración de los mismos con las propuestas virtuales de la universidad. En función de las opciones planteadas por Arroyo Vázquez y Gómez-Hernández (2020) y las propias percepciones de los bibliotecarios respecto a su integración con las propuestas virtuales de enseñanza aprendizaje, se puede afirmar que el $30 \%$ de las bibliotecas universitarias se corresponden a un "nivel de integración bajo o nulo"; un 54\% se vincula a un "nivel de integración intermedio" (reflejado por las respuestas "integración de recursos" "los bibliotecarios colaboramos...") y un 5\% responde a un "nivel de integración alto" (manifestado con la "integración de personal" en las propias aulas virtuales). De esta manera, los resultados se corresponden con las investigaciones precedentes que manifestaron que la opción intermedia es la que incluye a la mayoría de las bibliotecas universitarias.

\section{4 Identidad pospandemia}

Se visualiza una autopercepción de las bibliotecas universitarias como conocedoras de los ambientes mixtos de enseñanza, reconociéndose como un aliado importante del campus virtual, lo que podría resultar en un aspecto con gran potencial de crecimiento en un futuro. 
No obstante, si bien reconocen esta posibilidad, mencionan la existencia de desiguales condiciones para el paso siguiente. Mientras algunas proclamaron la oportunidad que plantea el contexto, otras manifestaron un estado de crisis; para algunas instituciones representa un entrenamiento para el cambio, otras debieron enfrentar resistencias.

Es destacable el marcado reconocimiento a la necesidad de evolución, manifestada por las expresiones de repensar, adaptarse, integrarse, cooperar, bregar por el conocimiento abierto, generar valor y atender a las nuevas necesidades.

\section{CONCLUSIONES}

Resulta pertinente recuperar aportes como el de Cruz Durañona y Marañón Rodríguez (2018) quienes previo a la pandemia y confinamiento COVID19 planteaban que las bibliotecas universitarias hoy se presentan como facilitadoras del proceso de aprendizaje continuo, que supone un cambio cultural, tanto de los usuarios como del personal bibliotecario.

Las bibliotecas universitarias, como muchos otros, han legitimado a la virtualidad. Ya no será posible pensar en un porfolio de servicios que no incluya a la capacitación de usuarios ni una comunidad de alcance meramente institucional o local.

¿COVID 19 convirtió al mundo en un ambiente VUCA? Según lo definido por Bennis y Nanus (1985) parecería que ahora vivimos una vida VUCA caracterizada por los atributos de: Volatility, Uncertainty, Complexity y Ambiguity (en español: Volatilidad, Incertidumbre, Complejidad y Ambigüedad). Este modelo que surgió para referir en el mundo de las empresas al estilo de liderazgo requerido para desenvolverse en ambientes donde el cambio es la constante, pospandemia podría aplicar a todos los sectores en que nos desenvolvemos las personas, y en los que las bibliotecas académicas no están exentas. Donde la realidad nos propone un mundo VUCA, necesitamos recuperar aquello que es sólido, cierto, simple y preciso.

El desafío en un futuro inmediato es superar la adaptación a la coyuntura que se hizo, y aprovechar la oportunidad de volver a pensar el ser Biblioteca Universitaria en el hoy. Una evolución que se ha vuelto irremediablemente necesaria.

\section{BIBLIOGRAFÍA}

ALONSO-ARÉVALO, J. Las bibliotecas universitarias ante un presente y un futuro inéditos. Texto del II FORO VIRTUAL DGB-UNAM. Nuevos desafios de las bibliotecas universitarias ante la COVID-19, 2020.

ARROYO-VÁZQUEZ, N. y GÓMEZ-HERNÁNDEZ, J. La biblioteca integrada en la enseñanza universitaria online: situación en España. El profesional de la información, 2020, vol. 29, nº 4, p.1-11.

ASOCIACIÓN COLOMBIANA DE UNIVERSIDADES [en línea]. Disponible en: $<$ https://www.ascun.org.co/uploads/default/news/53047ae8af2bb5c358843c886baa4447.pdf $>$ [Consulta: 6 de agosto de 2020]

ASSOCIATION OF COLLEGES \& RESEARCH LIBRARIES - AMERICAN LIBRARY ASSOCIATION [en línea]. Disponible en: $<$ http://www.ala.org/acrl/> [Consulta: 7 de octubre de 2020]

BENNIS, W.G. y NANUS, B. Leaders: The Strategies for taking charge. NewYork: Harper\& Row, Publishers. 1985.

BROWN, S.; BENNETT, C.; HENSON, B. y VALK, A. Next-gen learning spaces. Association of Research Libraries, 2014.

COALICIÓN INTERNACIONAL DE CONSORCIOS DE BIBLIOTECAS [en línea]. Disponible en: $<\mathrm{http}: / /$ www.icolc.net/> [Consulta: 20 de julio de 2020]

CONSEJO DE RECTORES DE UNIVERSIDADES CHILENAS. Comisión Asesora de Bibliotecas y Documentación. Estándares para bibliotecas universitarias chilenas, 2001.

CRUZ DURAÑONA, M. y MARAÑÓN RODRÍGUEZ, E. Estrategia para transformar la biblioteca Juan Cristobal Napoles Fajardo en un centro de recursos para el aprendizaje y la investigación que contribuya al desarrollo cultural de la comunidad de la Universidad de Las Tunas. Didasc@lia: Didáctica y Educación, 2018, vol. 9, n 5 abril (Edición especial), p. 193-218.

DE SILVA, M.B.; ORTEGA, E. y OCANDO, D. ¿Cuáles competencias en qué docente bibliotecario? Boletín de la Asociación Andaluza de Bibliotecarios, 2008, vol. 23, n 90, p. 55-70.

GALLO-LEÓN, J.P. La biblioteca es servicio (y en ello está nuestro futuro). El profesional de la información, 2015, vol. 24, no 2, p. 87-93.

REBIUN [en línea]. Disponible en: <https://www.rebiun.org/> [Consulta: 20 de julio de 2020]

RED AMICUS [en línea]. Disponible en: <https://www.udesa.edu.ar/amicus $>$ [Consulta: 20 de julio de 2020] 
RED INTERUNIVERSITARIA ARGENTINA DE BIBLIOTECAS. REDIAB [en línea]. Disponible en: $<$ http://www2.biblio.unlp.edu.ar/jubiuna $>$ [Consulta: 20 de julio de 2020]

UNESCO [en línea]. Disponible en: <https://es.unesco.org/covid19/educationresponse> [Consulta: 5 de agosto de 2020]

WATSON, L. El diseño de la biblioteca universitaria del siglo XXI: ideas y tendencias. BID: textos universitaris de biblioteconomía i documentació, 2017, n 38, p. 1-10. 